\title{
29. SEISMIC VELOCITIES, ELECTRICAL RESISTIVITIES, DENSITIES, AND POROSITIES OF BASALTS FROM DSDP LEG 46
}

\author{
N.I. Christensen, ${ }^{2}$ R.D. Hyndman, ${ }^{3}$ J.M. Hull, ${ }^{2}$ and M.H. Salisbury ${ }^{4}$
}

\section{INTRODUCTION}

Laboratory data on physical properties are particularly important for Leg 46 rocks because of the successful downhole logging program at Site 396B. The purposes of this investigation were to (1) measure velocities and elastic properties of basalts from Site 396B under conditions of confining pressure and water saturation appropriate to the sea floor for comparisons with the downhole velocity measurements; (2) measure electrical resistivities of saturated basalt samples for comparisons with the downhole resistivity measurements; and (3) determine bulk densities and porosities of the basalts for correlations with the other physical properties.

\section{EXPERIMENTAL TECHNIQUES AND DATA}

Compressional $\left(\mathrm{V}_{p}\right)$ and shear wave $\left(\mathrm{V}_{s}\right)$ velocities are given in Table 1, along with dry bulk densities $(\rho)$, wet bulk densities $\left(\rho^{*}\right)$, porosities $(\phi)$, ratios of compressional to shear velocities $\left(\mathrm{V}_{p} / \mathrm{V}_{s}\right)$, Poisson's ratios $(\sigma)$, bulk moduli $(K)$, shear moduli $(\mu)$, Lamés' constants $(\lambda)$, and Young's moduli $(E)$.

Cores $2.5 \mathrm{~cm}$ in diameter and 3 to $5 \mathrm{~cm}$ in length were used for the velocity measurements. Five of the 25 basalt samples were stored in seawater immediately after recovery on ship. After arrival in our laboratory, wet weights were measured for the saturated basalts, while the other samples were dried for two weeks. Dry weights were measured and the basalts were then water saturated and wet weights were obtained prior to the velocity measurements. The samples which were saturated on ship were dried for two weeks after the velocity measurements and then reweighed. The dry densities, wet densities, and porosities in Table 1 were calculated using the core volumes and the wet and dry weights.

Velocities were obtained from the travel times of elastic waves through samples of known length using the pulse transmission technique (Birch, 1960). Barium titanate and $\mathrm{AC}$-cut quartz transducers of 1 to $2 \mathrm{MHz}$ frequencies generated and received compressional and shear waves, respectively. Pore pressures were maintained at values lower than external pressures by placing 100 mesh screen between the samples and the

'Contribution of the Earth Physics Branch No. 672.

${ }^{2}$ Department of Geological Sciences, University of Washington, Seattle, Washington

${ }^{3}$ Pacific Geoscience Center, Earth Physics Branch, Department of Energy, Mines and Resources, Victoria, B.C. Canada.

${ }^{4}$ Scripps Institution of Oceanography, University of Calfornia San Diego, La Jolla, California. copper jackets. The transducers were coupled to the samples with rubber tubing and pressure was applied in $0.2-$ and $1.0-\mathrm{kbar}$ steps. The pressure-generating system contains a two-stage intensifier using a low-viscosity oil for the pressure medium. Pressure was measured with a calibrated manganin coil located on the high pressure side of the intensifier.

The velocities are estimated to be accurate to within 1 per cent (Christensen and Shaw, 1970). Elastic constants were calculated from the velocities and densities with the equations summarized by Birch (1961). Velocities and densities used for the calculations and given in Table 1 were corrected for compression using an iterative technique and the dynamically determined compressibilities.

The electrical resistivities of the $2.5-\mathrm{cm}$-diameter, 5cm-long basalt cores were measured by a simple two electrode technique at $22^{\circ} \mathrm{C}$ and atmospheric pressure (see Hyndman and Drury, 1977). A $0.5 \mathrm{~V}, 50 \mathrm{~Hz}$ signal was applied to the ends which had been painted with electrically conducting epoxy resin. The measurement precision is better than 1 per cent, but variations in the amount of surface drying make the values reproducible only to \pm 10 per cent (Table 2 ). All of the samples were saturated with sea water of resistivity about $0.2 \mathrm{ohm}-\mathrm{m}$ at $22^{\circ} \mathrm{C}$ on the assumption that this approximated their in situ state. Hyndman and Drury (1977) found that sea floor basalt resistivities increase by up to a factor of two in going from atmospheric pressure to an upper crustal pressure of $0.5 \mathrm{kbar}$, and decrease by about a factor of 2 for a temperature increase of $10^{\circ}$ to $20^{\circ} \mathrm{C}$. At depths penetrated by the borehole the temperature is slightly lower and the confining pressure greater than in the laboratory, so the in situ resistivities may be up to a factor of two lower than those measured.

\section{DISCUSSION}

The basalt samples for which velocities are reported were selected to be as free of fractures as possible. Velocities were not measured in the basalt sands, breccias, or palagonite-rich rocks. Thus the data in Table 1 provide upper bounds on velocities, densities, and porosities of the upper crust in the vicinity of Hole 396B. In comparison with basalts from many older DSDP sites in which the rocks have been highly altered by sea floor weathering (Christensen and Salisbury, 1975), the rocks from Hole 396B are remarkably uniform in velocities, densities, and porosities. The mean compressional wave velocity at $0.5 \mathrm{kbar}$ for the samples of Table 1 is $6.04 \mathrm{~km} / \mathrm{sec}$. As expected, the mean velocity is higher than the velocities obtained from the downhole logging measurements. 
TABLE 1

Velocities, Elastic Constants, Densities, and Porosities

\begin{tabular}{|c|c|c|c|c|c|c|c|c|c|}
\hline $\begin{array}{c}\text { Sample } \\
\text { (Interval in } \mathrm{cm} \text { ) }\end{array}$ & $\begin{array}{c}P \\
\text { (kbar) }\end{array}$ & $\begin{array}{c}V_{p} \\
(\mathrm{~km} / \mathrm{sec})\end{array}$ & $\begin{array}{c}V_{s} \\
(\mathrm{~km} / \mathrm{sec})\end{array}$ & $V_{p} / V_{s}$ & $\sigma$ & $\begin{array}{c}K \\
(\mathrm{Mb})\end{array}$ & $\stackrel{\mu}{\mathrm{Mb})}$ & $\stackrel{\lambda}{\lambda}$ & $\begin{array}{c}E \\
(\mathrm{Mb})\end{array}$ \\
\hline $\begin{array}{l}396 \mathrm{~B}-5-2,50-54 \\
\rho=2.70 \mathrm{~g} / \mathrm{cm}^{3} \\
\rho^{*}=2.78 \mathrm{~g} / \mathrm{cm}^{3} \\
\phi=6.3 \%\end{array}$ & $\begin{array}{l}0.2 \\
0.4 \\
0.6 \\
0.8 \\
1.0 \\
2.0 \\
4.0 \\
6.0\end{array}$ & $\begin{array}{l}6.05 \\
6.07 \\
6.09 \\
6.11 \\
6.12 \\
6.17 \\
6.23 \\
6.24\end{array}$ & $\begin{array}{l}3.16 \\
3.21 \\
3.25 \\
3.28 \\
3.30 \\
3.35 \\
3.40 \\
3.41\end{array}$ & $\begin{array}{l}1.91 \\
1.89 \\
1.87 \\
1.86 \\
1.85 \\
1.84 \\
1.83 \\
1.83\end{array}$ & $\begin{array}{l}0.31 \\
0.31 \\
0.30 \\
0.30 \\
0.30 \\
0.29 \\
0.29 \\
0.29\end{array}$ & $\begin{array}{l}0.63 \\
0.62 \\
0.62 \\
0.62 \\
0.62 \\
0.63 \\
0.63 \\
0.64\end{array}$ & $\begin{array}{l}0.27 \\
0.28 \\
0.28 \\
0.29 \\
0.29 \\
0.30 \\
0.31 \\
0.32\end{array}$ & $\begin{array}{l}0.45 \\
0.44 \\
0.43 \\
0.43 \\
0.42 \\
0.43 \\
0.42 \\
0.43\end{array}$ & $\begin{array}{l}0.71 \\
0.73 \\
0.74 \\
0.75 \\
0.76 \\
0.78 \\
0.81 \\
0.81\end{array}$ \\
\hline $\begin{array}{l}396 \mathrm{~B}-7-1,60-83 \\
\rho=2.78 \\
\rho^{*}=2.82 \\
\phi=3.9 \%\end{array}$ & $\begin{array}{l}0.2 \\
0.4 \\
0.6 \\
0.8 \\
1.0 \\
2.0 \\
4.0 \\
6.0\end{array}$ & $\begin{array}{l}5.93 \\
5.95 \\
5.97 \\
5.99 \\
6.00 \\
6.02 \\
6.09 \\
6.13\end{array}$ & $\begin{array}{l}3.16 \\
3.19 \\
3.21 \\
3.23 \\
3.25 \\
3.29 \\
3.32 \\
3.34\end{array}$ & $\begin{array}{l}1.88 \\
1.87 \\
1.86 \\
1.85 \\
1.85 \\
1.83 \\
1.83 \\
1.84\end{array}$ & $\begin{array}{l}0.30 \\
0.30 \\
0.30 \\
0.30 \\
0.29 \\
0.29 \\
0.29 \\
0.29\end{array}$ & $\begin{array}{l}0.61 \\
0.61 \\
0.61 \\
0.61 \\
0.61 \\
0.61 \\
0.63 \\
0.64\end{array}$ & $\begin{array}{l}0.28 \\
0.28 \\
0.29 \\
0.29 \\
0.29 \\
0.30 \\
0.31 \\
0.31\end{array}$ & $\begin{array}{l}0.42 \\
0.42 \\
0.42 \\
0.42 \\
0.41 \\
0.41 \\
0.42 \\
0.43\end{array}$ & $\begin{array}{l}0.72 \\
0.74 \\
0.74 \\
0.75 \\
0.76 \\
0.78 \\
0.80 \\
0.81\end{array}$ \\
\hline $\begin{array}{l}396 \mathrm{~B}-8-1,61-73 \\
\rho=2.83 \\
\rho^{*}=2.85 \\
\phi=2.0 \%\end{array}$ & $\begin{array}{l}0.2 \\
0.4 \\
0.6 \\
0.8 \\
1.0 \\
2.0 \\
4.0 \\
6.0\end{array}$ & $\begin{array}{l}6.11 \\
6.13 \\
6.15 \\
6.17 \\
6.18 \\
6.21 \\
6.26 \\
6.28\end{array}$ & $\begin{array}{l}3.27 \\
3.29 \\
3.30 \\
3.31 \\
3.32 \\
3.35 \\
3.38 \\
3.40\end{array}$ & $\begin{array}{l}1.87 \\
1.86 \\
1.86 \\
1.86 \\
1.86 \\
1.86 \\
1.85 \\
1.85\end{array}$ & $\begin{array}{l}0.30 \\
0.30 \\
0.30 \\
0.30 \\
0.30 \\
0.30 \\
0.29 \\
0.29\end{array}$ & $\begin{array}{l}0.65 \\
0.65 \\
0.66 \\
0.66 \\
0.66 \\
0.67 \\
0.68 \\
0.69\end{array}$ & $\begin{array}{l}0.30 \\
0.31 \\
0.31 \\
0.31 \\
0.31 \\
0.32 \\
0.33 \\
0.33\end{array}$ & $\begin{array}{l}0.45 \\
0.45 \\
0.45 \\
0.46 \\
0.46 \\
0.46 \\
0.46 \\
0.47\end{array}$ & $\begin{array}{l}0.79 \\
0.79 \\
0.80 \\
0.80 \\
0.81 \\
0.82 \\
0.84 \\
0.85\end{array}$ \\
\hline $\begin{array}{l}396 \mathrm{~B}-8-2,10-20 \\
\rho=2.71 \\
\rho^{*}=2.77 \\
\phi=6.0 \%\end{array}$ & $\begin{array}{l}0.2 \\
0.4 \\
0.6 \\
0.8 \\
1.0 \\
2.0 \\
4.0 \\
6.0\end{array}$ & $\begin{array}{l}5.92 \\
5.95 \\
5.97 \\
5.98 \\
6.00 \\
6.05 \\
6.14 \\
6.20\end{array}$ & $\begin{array}{l}3.13 \\
3.17 \\
3.19 \\
3.21 \\
3.23 \\
3.27 \\
3.31 \\
3.34\end{array}$ & $\begin{array}{l}1.89 \\
1.88 \\
1.87 \\
1.86 \\
1.86 \\
1.85 \\
1.85 \\
1.86\end{array}$ & $\begin{array}{l}0.31 \\
0.30 \\
0.30 \\
0.30 \\
0.30 \\
0.29 \\
0.29 \\
0.30\end{array}$ & $\begin{array}{l}0.60 \\
0.60 \\
0.60 \\
0.60 \\
0.60 \\
0.61 \\
0.63 \\
0.65\end{array}$ & $\begin{array}{l}0.27 \\
0.27 \\
0.28 \\
0.28 \\
0.28 \\
0.29 \\
0.30 \\
0.31\end{array}$ & $\begin{array}{l}0.42 \\
0.42 \\
0.41 \\
0.41 \\
0.41 \\
0.42 \\
0.43 \\
0.44\end{array}$ & $\begin{array}{l}0.69 \\
0.71 \\
0.72 \\
0.73 \\
0.73 \\
0.75 \\
0.78 \\
0.79\end{array}$ \\
\hline $\begin{array}{l}369 \mathrm{~B}-9-1,89-93 \\
\rho=2.77 \\
\rho^{*}=2.81 \\
\phi=3.2 \%\end{array}$ & $\begin{array}{l}0.2 \\
0.4 \\
0.6 \\
0.8 \\
1.0 \\
2.0 \\
4.0 \\
6.0\end{array}$ & $\begin{array}{l}5.90 \\
5.93 \\
5.94 \\
5.96 \\
5.97 \\
6.00 \\
6.07 \\
6.10\end{array}$ & $\begin{array}{l}3.18 \\
3.18 \\
3.19 \\
3.19 \\
3.20 \\
3.22 \\
3.24 \\
3.26\end{array}$ & $\begin{array}{l}1.86 \\
1.86 \\
1.86 \\
1.87 \\
1.87 \\
1.87 \\
1.87 \\
1.87\end{array}$ & $\begin{array}{l}0.30 \\
0.30 \\
0.30 \\
0.30 \\
0.30 \\
0.30 \\
0.30 \\
0.30\end{array}$ & $\begin{array}{l}0.59 \\
0.60 \\
0.60 \\
0.61 \\
0.61 \\
0.62 \\
0.64 \\
0.65\end{array}$ & $\begin{array}{l}0.28 \\
0.28 \\
0.28 \\
0.28 \\
0.28 \\
0.29 \\
0.29 \\
0.30\end{array}$ & $\begin{array}{l}0.40 \\
0.41 \\
0.41 \\
0.42 \\
0.42 \\
0.43 \\
0.44 \\
0.45\end{array}$ & $\begin{array}{l}0.73 \\
0.73 \\
0.73 \\
0.73 \\
0.74 \\
0.75 \\
0.76 \\
0.77\end{array}$ \\
\hline $\begin{array}{l}396 \mathrm{~B}-10-2,22-28 \\
\rho=2.79 \\
\rho^{*}=2.83 \\
\phi=3.5 \%\end{array}$ & $\begin{array}{l}0.2 \\
0.4 \\
0.6 \\
0.8 \\
1.0 \\
2.0 \\
4.0 \\
6.0\end{array}$ & $\begin{array}{l}6.18 \\
6.21 \\
6.23 \\
6.26 \\
6.28 \\
6.34 \\
6.42 \\
6.45\end{array}$ & $\begin{array}{l}3.20 \\
3.29 \\
3.33 \\
3.36 \\
3.38 \\
3.45 \\
3.50 \\
3.52\end{array}$ & $\begin{array}{l}1.93 \\
1.89 \\
1.87 \\
1.86 \\
1.86 \\
1.84 \\
1.83 \\
1.83\end{array}$ & $\begin{array}{l}0.32 \\
0.30 \\
0.30 \\
0.30 \\
0.30 \\
0.29 \\
0.29 \\
0.29\end{array}$ & $\begin{array}{l}0.68 \\
0.67 \\
0.67 \\
0.67 \\
0.68 \\
0.68 \\
0.70 \\
0.71\end{array}$ & $\begin{array}{l}0.29 \\
0.30 \\
0.31 \\
0.32 \\
0.32 \\
0.33 \\
0.34 \\
0.35\end{array}$ & $\begin{array}{l}0.49 \\
0.47 \\
0.46 \\
0.46 \\
0.46 \\
0.46 \\
0.47 \\
0.47\end{array}$ & $\begin{array}{l}0.75 \\
0.79 \\
0.80 \\
0.82 \\
0.83 \\
0.86 \\
0.89 \\
0.90\end{array}$ \\
\hline $\begin{array}{l}396 \mathrm{~B}-12-1,59-93 \\
\rho=2.82 \\
\rho^{*}=2.84 \\
\phi=2.4 \%\end{array}$ & $\begin{array}{l}0.2 \\
0.4 \\
0.6 \\
0.8 \\
1.0 \\
2.0 \\
4.0 \\
6.0\end{array}$ & $\begin{array}{l}5.85 \\
5.87 \\
5.90 \\
5.92 \\
5.93 \\
6.00 \\
6.08 \\
6.12\end{array}$ & $\begin{array}{l}3.31 \\
3.32 \\
3.33 \\
3.33 \\
3.33 \\
3.34 \\
3.34 \\
3.36\end{array}$ & $\begin{array}{l}1.77 \\
1.77 \\
1.77 \\
1.78 \\
1.78 \\
1.80 \\
1.82 \\
1.82\end{array}$ & $\begin{array}{l}0.26 \\
0.26 \\
0.27 \\
0.27 \\
0.27 \\
0.28 \\
0.28 \\
0.28\end{array}$ & $\begin{array}{l}0.55 \\
0.56 \\
0.56 \\
0.57 \\
0.57 \\
0.60 \\
0.63 \\
0.64\end{array}$ & $\begin{array}{l}0.31 \\
0.31 \\
0.31 \\
0.31 \\
0.31 \\
0.31 \\
0.32 \\
0.32\end{array}$ & $\begin{array}{l}0.35 \\
0.35 \\
0.36 \\
0.36 \\
0.37 \\
0.39 \\
0.41 \\
0.42\end{array}$ & $\begin{array}{l}0.78 \\
0.79 \\
0.79 \\
0.79 \\
0.79 \\
0.80 \\
0.81 \\
0.83\end{array}$ \\
\hline $\begin{array}{l}396 \mathrm{~B}-13-1,55-60 \\
\rho=2.81 \\
\rho^{*}=2.84 \\
\phi=3.4 \%\end{array}$ & $\begin{array}{l}0.2 \\
0.4 \\
0.6 \\
0.8 \\
1.0 \\
2.0 \\
4.0 \\
6.0\end{array}$ & $\begin{array}{l}6.10 \\
6.12 \\
6.13 \\
6.15 \\
6.16 \\
6.21 \\
6.29 \\
6.34\end{array}$ & $\begin{array}{l}3.30 \\
3.32 \\
3.33 \\
3.34 \\
3.35 \\
3.39 \\
3.43 \\
3.45\end{array}$ & $\begin{array}{l}1.85 \\
1.84 \\
1.84 \\
1.84 \\
1.84 \\
1.83 \\
1.83 \\
1.84\end{array}$ & $\begin{array}{l}0.29 \\
0.29 \\
0.29 \\
0.29 \\
0.29 \\
0.29 \\
0.29 \\
0.29\end{array}$ & $\begin{array}{l}0.64 \\
0.64 \\
0.64 \\
0.64 \\
0.64 \\
0.66 \\
0.67 \\
0.69\end{array}$ & $\begin{array}{l}0.31 \\
0.31 \\
0.31 \\
0.31 \\
0.31 \\
0.32 \\
0.33 \\
0.34\end{array}$ & $\begin{array}{l}0.43 \\
0.43 \\
0.43 \\
0.44 \\
0.44 \\
0.44 \\
0.45 \\
0.46\end{array}$ & $\begin{array}{l}0.79 \\
0.80 \\
0.80 \\
0.81 \\
0.81 \\
0.83 \\
0.86 \\
0.87\end{array}$ \\
\hline
\end{tabular}


TABLE 1-Continued

\begin{tabular}{|c|c|c|c|c|c|c|c|c|c|}
\hline $\begin{array}{c}\text { Sample } \\
\text { (Interval in } \mathrm{cm} \text { ) }\end{array}$ & $\begin{array}{c}P \\
\text { (kbar) }\end{array}$ & $\begin{array}{c}V_{p} \\
(\mathrm{~km} / \mathrm{sec})\end{array}$ & $\begin{array}{c}V_{s} \\
(\mathrm{~km} / \mathrm{sec})\end{array}$ & $V_{p} / V_{s}$ & $\sigma$ & $\begin{array}{c}K \\
(\mathrm{Mb})\end{array}$ & $\stackrel{\mu}{(\mathrm{Mb})}$ & $\stackrel{\lambda}{(\mathrm{Mb})}$ & $\begin{array}{c}E \\
(\mathrm{Mb})\end{array}$ \\
\hline 396B-14-2, 39-45 & 0.2 & 6.24 & 3.29 & 1.90 & 0.31 & 0.70 & 0.31 & 0.49 & 0.81 \\
\hline$\rho=2.86$ & 0.4 & $\cdot 6.28$ & 3.30 & 1.90 & 0.31 & 0.71 & 0.31 & 0.50 & 0.82 \\
\hline$\rho^{*}=2.87$ & 0.6 & 6.30 & 3.31 & 1.90 & 0.31 & 0.72 & 0.31 & 0.51 & 0.82 \\
\hline \multirow[t]{5}{*}{$\phi=1.4 \%$} & 0.8 & 6.31 & 3.32 & 1.90 & 0.31 & 0.72 & 0.32 & 0.51 & 0.82 \\
\hline & 1.0 & 6.32 & 3.33 & 1.90 & 0.31 & 0.72 & 0.32 & 0.51 & 0.83 \\
\hline & 2.0 & 6.35 & 3.36 & 1.89 & 0.31 & 0.73 & 0.32 & 0.51 & 0.84 \\
\hline & 4.0 & 6.41 & 3.39 & 1.89 & 0.31 & 0.74 & 0.33 & 0.52 & 0.86 \\
\hline & 6.0 & 6.45 & 3.41 & 1.89 & 0.31 & 0.75 & 0.34 & 0.53 & 0.88 \\
\hline 396B-15-1,? & 0.2 & 6.07 & 3.29 & 1.84 & 0.29 & 0.64 & 0.31 & 0.43 & 0.80 \\
\hline$\rho=2.86$ & 0.4 & 6.12 & 3.31 & 1.85 & 0.29 & 0.65 & 0.31 & 0.44 & 0.81 \\
\hline$\rho *=2.88$ & 0.6 & 6.15 & 3.32 & 1.85 & 0.29 & 0.66 & 0.32 & 0.45 & 0.82 \\
\hline \multirow{5}{*}{$\phi=2.0 \%$} & 0.8 & 6.17 & 3.33 & 1.85 & 0.29 & 0.67 & 0.32 & 0.45 & 0.82 \\
\hline & 1.0 & 6.19 & 3.34 & 1.85 & 0.29 & 0.67 & 0.32 & 0.46 & 0.83 \\
\hline & -2.0 & 6.25 & 3.37 & 1.86 & 0.30 & 0.69 & 0.32 & 0.47 & 0.84 \\
\hline & 4.0 & 6.34 & 3.38 & 1.87 & 0.30 & 0.72 & 0.33 & 0.50 & 0.86 \\
\hline & 6.0 & 6.38 & 3.38 & 1.89 & 0.31 & 0.73 & 0.33 & 0.51 & 0.86 \\
\hline $396 \mathrm{~B}-15-3,68-75$ & 0.2 & 5.78 & 3.12 & 1.85 & 0.29 & 0.56 & 0.27 & 0.38 & 0.69 \\
\hline$\rho=2.75$ & 0.4 & 5.81 & 3.13 & 1.86 & 0.30 & 0.57 & 0.27 & 0.39 & 0.70 \\
\hline$\rho^{*}=2.78$ & 0.6 & 5.84 & 3.15 & 1.85 & 0.29 & 0.57 & 0.27 & 0.39 & 0.71 \\
\hline \multirow{5}{*}{$\phi=3.5 \%$} & 0.8 & 5.86 & 3.16 & 1.85 & 0.29 & 0.58 & 0.27 & 0.39 & 0.71 \\
\hline & 1.0 & 5.88 & 3.17 & 1.85 & 0.30 & 0.58 & 0.28 & 0.40 & 0.72 \\
\hline & 2.0 & 5.94 & 3.20 & 1.86 & 0.30 & 0.59 & 0.28 & 0.41 & 0.73 \\
\hline & 4.0 & 6.02 & 3.24 & 1.86 & 0.30 & 0.61 & 0.29 & 0.42 & 0.75 \\
\hline & 6.0 & 6.09 & 3.26 & 1.87 & 0.30 & 0.64 & 0.29 & 0.44 & 0.77 \\
\hline 396B-16-1, 73-82 & 0.2 & 6.25 & 3.38 & 1.85 & 0.29 & 0.67 & 0.32 & 0.46 & 0.83 \\
\hline$\rho=2.81$ & 0.4 & 6.26 & 3.37 & 1.86 & 0.30 & 0.67 & 0.32 & 0.46 & 0.83 \\
\hline$\rho^{*}=2.84$ & 0.6 & 6.28 & 3.38 & 1.86 & 0.30 & 0.68 & 0.32 & 0.47 & 0.83 \\
\hline \multirow[t]{5}{*}{$\phi=2.9 \%$} & 0.8 & 6.29 & 3.39 & 1.86 & 0.30 & 0.68 & 0.32 & 0.47 & 0.84 \\
\hline & 1.0 & 6.30 & 3.40 & 1.85 & 0.29 & 0.68 & 0.32 & 0.47 & 0.84 \\
\hline & 2.0 & 6.31 & 3.43 & 1.84 & 0.29 & 0.68 & 0.33 & 0.46 & 0.85 \\
\hline & 4.0 & 6.36 & 3.46 & 1.84 & 0.29 & 0.69 & 0.34 & 0.46 & 0.87 \\
\hline & 6.0 & 6.38 & 3.48 & 1.89 & 0.31 & 0.72 & 0.32 & 0.51 & 0.84 \\
\hline $396 \mathrm{~B}-16-4,127-139$ & 0.2 & 6.21 & 3.39 & 1.83 & 0.29 & 0.66 & 0.33 & 0.44 & 0.84 \\
\hline$\rho=2.83$ & 0.4 & 6.24 & 3.40 & 1.84 & 0.29 & 0.67 & 0.33 & 0.45 & 0.84 \\
\hline$\rho^{*}=2.84$ & 0.6 & 6.27 & 3.41 & 1.84 & 0.29 & 0.67 & 0.33 & 0.45 & 0.85 \\
\hline \multirow[t]{5}{*}{$\phi=1.0 \%$} & 0.8 & 6.29 & 3.42 & 1.84 & 0.29 & 0.68 & 0.33 & 0.46 & 0.85 \\
\hline & 1.0 & 6.31 & 3.43 & 1.84 & 0.29 & 0.68 & 0.33 & 0.46 & 0.86 \\
\hline & 2.0 & 6.38 & 3.46 & 1.85 & 0.29 & 0.70 & 0.34 & 0.48 & 0.88 \\
\hline & 4.0 & 6.47 & 3.47 & 1.86 & 0.30 & 0.73 & 0.34 & 0.50 & 0.89 \\
\hline & 6.0 & 6.50 & 3.49 & 1.86 & 0.30 & 0.74 & 0.35 & 0.51 & 0.90 \\
\hline $396 \mathrm{~B}-17-3,20-30$ & 0.2 & 6.20 & 3.27 & 1.90 & 0.31 & 0.68 & 0.30 & 0.48 & 0.79 \\
\hline$\rho=2.83$ & 0.4 & 6.24 & 3.28 & 1.90 & 0.31 & 0.70 & 0.30 & 0.49 & 0.80 \\
\hline$\rho^{*}=2.85$ & 0.6 & 6.26 & 3.30 & 1.90 & 0.31 & 0.70 & 0.31 & 0.49 & 0.81 \\
\hline \multirow[t]{5}{*}{$\phi=1.9 \%$} & 0.8 & 6.28 & 3.31 & 1.90 & 0.31 & 0.70 & 0.31 & 0.50 & 0.81 \\
\hline & 1.0 & 6.29 & 3.32 & 1.89 & 0.31 & 0.70 & 0.31 & 0.50 & 0.81 \\
\hline & 2.0 & 6.33 & 3.35 & 1.89 & 0.31 & 0.71 & 0.32 & 0.50 & 0.83 \\
\hline & 4.0 & 6.39 & 3.38 & 1.89 & 0.31 & 0.73 & 0.33 & 0.51 & 0.85 \\
\hline & 6.0 & 6.42 & 3.40 & 1.89 & 0.31 & 0.74 & 0.33 & 0.52 & 0.86 \\
\hline $396 \mathrm{~B}-18-2,52-55$ & 0.2 & 5.50 & 2.84 & 1.94 & 0.32 & 0.51 & 0.21 & 0.37 & 0.55 \\
\hline$\rho=2.60$ & 0.4 & 5.56 & 2.99 & 1.86 & 0.30 & 0.49 & 0.23 & 0.34 & 0.60 \\
\hline$\rho^{*}=2.70$ & 0.6 & 5.60 & 3.05 & 1.84 & 0.29 & 0.49 & 0.24 & 0.33 & 0.62 \\
\hline \multirow[t]{5}{*}{$\phi=9.9 \%$} & 0.8 & 5.64 & 3.08 & 1.83 & 0.29 & 0.50 & 0.25 & 0.33 & 0.64 \\
\hline & 1.0 & 5.66 & 3.11 & 1.82 & 0.28 & 0.50 & 0.25 & 0.33 & 0.65 \\
\hline & 2.0 & 5.75 & 3.20 & 1.80 & 0.28 & 0.51 & 0.27 & 0.33 & 0.68 \\
\hline & 4.0 & 5.85 & 3.29 & 1.78 & 0.27 & 0.52 & 0.28 & 0.33 & 0.72 \\
\hline & 6.0 & 5.92 & 3.31 & 1.79 & 0.27 & 0.54 & 0.29 & 0.35 & 0.73 \\
\hline $396 \mathrm{~B}-20-1,2-18$ & 0.2 & 6.19 & 3.09 & 2.00 & 0.33 & 0.72 & 0.27 & 0.54 & 0.71 \\
\hline$\rho=2.81$ & 0.4 & 6.21 & 3.10 & 2.00 & 0.33 & 0.72 & 0.27 & 0.54 & 0.72 \\
\hline$\rho^{*}=2.82$ & 0.6 & 6.23 & 3.11 & 2.00 & 0.33 & 0.73 & 0.27 & 0.55 & 0.72 \\
\hline \multirow[t]{5}{*}{$\phi=1.2 \%$} & 0.8 & 6.25 & 3.11 & 2.01 & 0.34 & 0.73 & 0.27 & 0.55 & 0.73 \\
\hline & 1.0 & 6.26 & 3.12 & 2.01 & 0.33 & 0.74 & 0.27 & 0.55 & 0.73 \\
\hline & 2.0 & 6.32 & 3.14 & 2.02 & 0.34 & 0.76 & 0.28 & 0.57 & 0.74 \\
\hline & 4.0 & 6.42 & 3.16 & 2.03 & 0.34 & 0.79 & 0.28 & 0.60 & 0.76 \\
\hline & 6.0 & 6.46 & 3.17 & 2.04 & 0.34 & 0.80 & 0.28 & 0.61 & 0.76 \\
\hline
\end{tabular}


TABLE 1-Continued

\begin{tabular}{|c|c|c|c|c|c|c|c|c|c|}
\hline $\begin{array}{c}\text { Sample } \\
\text { (Interval in } \mathrm{cm} \text { ) }\end{array}$ & $\begin{array}{c}P \\
\text { (kbar) }\end{array}$ & $\begin{array}{c}V_{p} \\
(\mathrm{~km} / \mathrm{sec})\end{array}$ & $\begin{array}{c}V_{S} \\
(\mathrm{~km} / \mathrm{sec})\end{array}$ & $V_{p} / V_{s}$ & $\sigma$ & $\begin{array}{c}K \\
(\mathrm{Mb})\end{array}$ & $\stackrel{\mu}{(\mathrm{Mb})}$ & $\stackrel{\lambda}{(\mathrm{Mb})}$ & $\begin{array}{c}E \\
(\mathrm{Mb})\end{array}$ \\
\hline $396 \mathrm{~B}-20-2,21-30$ & 0.2 & 5.45 & 2.74 & 1.99 & 0.33 & 0.51 & 0.19 & 0.38 & 0.52 \\
\hline$\rho=2.60$ & 0.4 & 5.49 & 2.79 & 1.97 & 0.33 & 0.51 & 0.20 & 0.38 & 0.54 \\
\hline$\rho^{*}=2.67$ & 0.6 & 5.52 & 2.82 & 1.96 & 0.32 & 0.52 & 0.21 & 0.38 & 0.55 \\
\hline \multirow{5}{*}{$\phi=7.4 \%$} & 0.8 & 5.54 & 2.86 & 1.94 & 0.32 & 0.51 & 0.21 & 0.37 & 0.56 \\
\hline & 1.0 & 5.56 & 2.88 & 1.93 & 0.32 & 0.52 & 0.22 & 0.37 & 0.57 \\
\hline & 2.0 & 5.64 & 2.97 & 1.90 & 0.31 & 0.52 & 0.23 & 0.37 & 0.60 \\
\hline & 4.0 & 5.75 & 3.04 & 1.89 & 0.31 & 0.54 & 0.24 & 0.38 & 0.63 \\
\hline & 6.0 & 5.83 & 3.09 & 1.89 & 0.30 & 0.56 & 0.25 & 0.39 & 0.65 \\
\hline $396 \mathrm{~B}-20-5,94-102$ & 0.2 & 6.13 & 3.19 & 1.92 & 0.31 & 0.66 & 0.28 & 0.48 & 0.74 \\
\hline$\rho=2.77$ & 0.4 & 6.19 & 3.22 & 1.92 & 0.31 & 0.68 & 0.29 & 0.49 & 0.75 \\
\hline$\rho^{*}=2.78$ & 0.6 & 6.22 & 3.25 & 1.91 & 0.31 & 0.68 & 0.29 & 0.49 & 0.77 \\
\hline \multirow[t]{5}{*}{$\phi=1.7 \%$} & 0.8 & 6.25 & 3.27 & 1.91 & 0.31 & 0.69 & 0.30 & 0.49 & 0.78 \\
\hline & 1.0 & 6.27 & 3.29 & 1.91 & 0.31 & 0.69 & 0.30 & 0.49 & 0.79 \\
\hline & 2.0 & 6.32 & 3.33 & 1.90 & 0.31 & 0.70 & 0.31 & 0.50 & 0.80 \\
\hline & 4.0 & 6.40 & 3.36 & 1.90 & 0.31 & 0.72 & 0.31 & 0.51 & 0.82 \\
\hline & 6.0 & 6.46 & 3.37 & 1.92 & 0.31 & 0.74 & 0.32 & 0.53 & 0.83 \\
\hline $396 \mathrm{~B}-20-6,43-53$ & 0.2 & 6.04 & 3.18 & 1.90 & 0.31 & 0.64 & 0.28 & 0.45 & 0.73 \\
\hline$\rho=2.76$ & 0.4 & 6.07 & 3.20 & 1.90 & 0.31 & 0.64 & 0.28 & 0.45 & 0.74 \\
\hline$\rho^{*}=2.79$ & 0.6 & 6.10 & 3.22 & 1.89 & 0.31 & 0.65 & 0.29 & 0.46 & 0.75 \\
\hline \multirow[t]{5}{*}{$\phi=3.1 \%$} & 0.8 & 6.13 & 3.23 & 1.90 & 0.31 & 0.65 & 0.29 & 0.46 & 0.75 \\
\hline & 1.0 & 6.15 & 3.24 & 1.90 & 0.31 & 0.66 & 0.29 & 0.47 & 0.76 \\
\hline & 2.0 & 6.21 & 3.26 & 1.90 & 0.31 & 0.68 & 0.30 & 0.48 & 0.77 \\
\hline & 4.0 & 6.30 & 3.29 & 1.91 & 0.31 & 0.70 & 0.30 & 0.50 & 0.79 \\
\hline & 6.0 & 6.39 & 3.32 & 1.92 & 0.31 & 0.72 & 0.31 & 0.52 & 0.81 \\
\hline $396 \mathrm{~B}-21-1,32-40$ & 0.2 & 6.16 & 3.34 & 1.84 & 0.29 & 0.65 & 0.31 & 0.44 & 0.81 \\
\hline$\rho=2.81$ & 0.4 & 6.18 & 3.35 & 1.84 & 0.29 & 0.65 & 0.31 & 0.44 & 0.81 \\
\hline$\rho^{*}=2.83$ & 0.6 & 6.19 & 3.36 & 1.84 & 0.29 & 0.65 & 0.32 & 0.44 & 0.82 \\
\hline \multirow{5}{*}{$\phi=2.8 \%$} & 0.8 & 6.21 & 3.37 & 1.84 & 0.29 & 0.66 & 0.32 & 0.44 & 0.82 \\
\hline & 1.0 & 6.22 & 3.38 & 1.84 & 0.29 & 0.66 & 0.32 & 0.44 & 0.83 \\
\hline & 2.0 & 6.26 & 3.41 & 1.84 & 0.29 & 0.67 & 0.33 & 0.45 & 0.84 \\
\hline & 4.0 & 6.34 & 3.43 & 1.85 & 0.29 & 0.69 & 0.33 & 0.47 & 0.86 \\
\hline & 6.0 & 6.38 & 3.45 & 1.85 & 0.29 & 0.70 & 0.34 & 0.48 & 0.87 \\
\hline $396 \mathrm{~B}-21-2,2-14$ & 0.2 & 5.84 & 2.81 & 2.08 & 0.35 & 0.64 & 0.21 & 0.50 & 0.58 \\
\hline$\rho=2.72$ & 0.4 & 5.88 & 2.82 & 2.09 & 0.35 & 0.65 & 0.22 & 0.51 & 0.58 \\
\hline$\rho^{*}=2.74$ & 0.6 & 5.90 & 2.83 & 2.08 & 0.35 & 0.66 & 0.22 & 0.51 & 0.59 \\
\hline \multirow[t]{5}{*}{$\phi=2.2 \%$} & 0.8 & 5.93 & 2.84 & 2.09 & 0.35 & 0.66 & 0.22 & 0.52 & 0.59 \\
\hline & 1.0 & 5.95 & 2.84 & 2.10 & 0.35 & 0.67 & 0.22 & 0.52 & 0.59 \\
\hline & 2.0 & 6.00 & 2.87 & 2.09 & 0.35 & 0.68 & 0.22 & 0.53 & 0.61 \\
\hline & 4.0 & 6.09 & 2.88 & 2.12 & 0.36 & 0.71 & 0.23 & 0.56 & 0.61 \\
\hline & 6.0 & 6.14 & 2.88 & 2.13 & 0.36 & 0.73 & 0.23 & 0.58 & 0.62 \\
\hline $396 \mathrm{~B}-22-2,40-54$ & 0.2 & 6.18 & 3.25 & 1.90 & 0.31 & 0.68 & 0.30 & 0.48 & 0.78 \\
\hline$\rho=2.82$ & 0.4 & 6.22 & 3.26 & 1.91 & 0.31 & 0.69 & 0.30 & 0.49 & 0.78 \\
\hline$\rho^{*}=2.84$ & 0.6 & 6.24 & 3.27 & 1.91 & 0.31 & 0.70 & 0.30 & 0.49 & 0.79 \\
\hline \multirow[t]{5}{*}{$\phi=2.4 \%$} & 0.8 & 6.26 & 3.28 & 1.91 & 0.31 & 0.70 & 0.30 & 0.50 & 0.79 \\
\hline & 1.0 & 6.28 & 3.29 & 1.91 & 0.31 & 0.70 & 0.30 & 0.50 & 0.80 \\
\hline & 2.0 & 6.33 & 3.32 & 1.91 & 0.31 & 0.72 & 0.31 & 0.51 & 0.81 \\
\hline & 4.0 & 6.42 & 3.35 & 1.91 & 0.31 & 0.74 & 0.32 & 0.53 & 0.84 \\
\hline & 6.0 & 6.48 & 3.38 & 1.92 & 0.31 & 0.76 & 0.32 & 0.54 & 0.85 \\
\hline $396 \mathrm{~B}-22-4,10-16$ & 0.2 & 6.00 & 3.17 & 1.89 & 0.31 & 0.62 & 0.28 & 0.44 & 0.73 \\
\hline$\rho=2.76$ & 0.4 & 6.05 & 3.19 & 1.90 & 0.31 & 0.64 & 0.28 & 0.45 & 0.74 \\
\hline$\rho^{*}=2.80$ & 0.6 & 6.09 & 3.21 & 1.90 & 0.31 & 0.65 & 0.28 & 0.46 & 0.74 \\
\hline \multirow{5}{*}{$\phi=3.6 \%$} & 0.8 & 6.11 & 3.22 & 1.90 & 0.31 & 0.65 & 0.29 & 0.46 & 0.75 \\
\hline & 1.0 & 6.14 & 3.24 & 1.90 & 0.31 & 0.66 & 0.29 & 0.46 & 0.76 \\
\hline & 2.0 & 6.20 & 3.28 & 1.89 & 0.31 & 0.67 & 0.30 & 0.47 & 0.78 \\
\hline & 4.0 & 6.29 & 3.31 & 1.90 & 0.31 & 0.69 & 0.31 & 0.49 & 0.80 \\
\hline & 6.0 & 6.38 & 3.35 & 1.90 & 0.31 & 0.72 & 0.31 & 0.51 & 0.82 \\
\hline $396 \mathrm{~B}-23-1,79-83$ & 0.2 & 5.80 & 2.80 & 2.07 & 0.35 & 0.61 & 0.21 & 0.47 & 0.56 \\
\hline$\rho=2.63$ & 0.4 & 5.85 & 2.82 & 2.07 & 0.35 & 0.62 & 0.21 & 0.48 & 0.56 \\
\hline$\rho^{*}=2.71$ & 0.6 & 5.89 & 2.84 & 2.07 & 0.35 & 0.63 & 0.21 & 0.49 & 0.57 \\
\hline \multirow{5}{*}{$\phi=8.3 \%$} & 0.8 & 5.93 & 2.86 & 2.07 & 0.35 & 0.64 & 0.21 & 0.49 & 0.58 \\
\hline & 1.0 & 5.95 & 2.87 & 2.07 & 0.35 & 0.64 & 0.22 & 0.50 & 0.58 \\
\hline & 2.0 & 6.05 & 2.93 & 2.07 & 0.35 & 0.66 & 0.23 & 0.51 & 0.61 \\
\hline & 4.0 & 6.19 & 2.98 & 2.07 & 0.35 & 0.70 & 0.24 & 0.54 & 0.63 \\
\hline & 6.0 & 6.29 & 3.00 & 2.10 & 0.35 & 0.73 & 0.24 & 0.57 & 0.65 \\
\hline
\end{tabular}


TABLE 1-Continued

\begin{tabular}{lccccccccc}
\hline $\begin{array}{c}\text { Sample } \\
\text { (Interval in cm) }\end{array}$ & $\begin{array}{c}P \\
(\mathrm{kbar})\end{array}$ & $\begin{array}{c}V_{p} \\
(\mathrm{~km} / \mathrm{sec})\end{array}$ & $\begin{array}{c}V_{s} \\
(\mathrm{~km} / \mathrm{sec})\end{array}$ & $V_{p} / V_{s}$ & $\sigma$ & $\begin{array}{c}K \\
(\mathrm{Mb})\end{array}$ & $\begin{array}{c}\mu \\
(\mathrm{Mb})\end{array}$ & $\begin{array}{c}\lambda \\
(\mathrm{Mb})\end{array}$ & $\begin{array}{c}E \\
(\mathrm{Mb})\end{array}$ \\
\hline $396 \mathrm{~B}-28-1,6-15$ & 0.2 & 5.68 & 2.97 & 1.91 & 0.31 & 0.56 & 0.24 & 0.40 & 0.63 \\
$\rho=2.72$ & 0.4 & 5.74 & 2.99 & 1.92 & 0.31 & 0.57 & 0.24 & 0.41 & 0.64 \\
$\rho^{*=2.77}$ & 0.6 & 5.78 & 3.01 & 1.92 & 0.31 & 0.58 & 0.25 & 0.42 & 0.65 \\
$\phi=5.0 \%$ & 0.8 & 5.81 & 3.03 & 1.92 & 0.31 & 0.59 & 0.25 & 0.42 & 0.66 \\
& 1.0 & 5.84 & 3.04 & 1.92 & 0.31 & 0.59 & 0.25 & 0.43 & 0.66 \\
& 2.0 & 5.93 & 3.09 & 1.92 & 0.31 & 0.61 & 0.26 & 0.44 & 0.68 \\
& 4.0 & 6.06 & 3.12 & 1.94 & 0.32 & 0.65 & 0.27 & 0.47 & 0.70 \\
& 6.0 & 6.17 & 3.14 & 1.97 & 0.33 & 0.68 & 0.27 & 0.50 & 0.72 \\
\hline
\end{tabular}

TABLE 2

DSDP Basalt Resistivities-Hole 396B

\begin{tabular}{lccc}
\hline $\begin{array}{c}\text { Sample } \\
\text { (Interval in cm) }\end{array}$ & $\begin{array}{c}\text { Resistivity } \\
\text { (ohm-m) }\end{array}$ & $\begin{array}{c}\text { Porosity } \\
(\% \text { vol) }\end{array}$ & \\
\hline $5-1,29-37$ & 79.3 & & \\
$5-1,57-68$ & 261 & & Measured at \\
$5-2,50-55$ & 166 & 6.3 & 0.5 V $50 \mathrm{~Hz}$ \\
$7-1,60-82$ & 279 & 3.9 & $22^{\circ} \mathrm{C}$ \\
$10-2,28-33$ & 351 & 3.5 & Atmospheric pressure \\
$14-1,97-108$ & 414 & & \\
$15-1,96-103$ & 411 & 2.0 & Seawater saturated \\
$15-2,116-120$ & 759 & & \\
$16-5,102-112$ & 432 & & \\
$17-2,80-90$ & 134 & & \\
$17-3,20-30$ & 557 & 1.9 & \\
$18-2,52-55$ & 55.5 & 9.9 & \\
$20-1,2-12$ & 627 & 1.2 & \\
$20-2,20-30$ & 36.5 & 7.4 & \\
$20-4,50-60$ & 178 & & \\
$20-6,43-53$ & 180 & 3.1 & \\
$21-4,32-40$ & 189 & 2.8 & \\
$22-1,122-140$ & 148 & & \\
$22-4,10-16$ & 176 & 3.6 & \\
$28-1,6-15$ & 91.6 & 5.0 & \\
\hline
\end{tabular}

Note: Geometric mean resistivity $215 \pm 40 \mathrm{ohm}$-m; geometric mean samples with porosity $203 \mathrm{ohm}-\mathrm{m}$; arithmetic mean porosity $4.2 \pm 7 \%$ vol; (geometric mean porosity $3.6 \%$ ).

Laboratory measurements of velocities in basalts from several relatively old DSDP sites have found seismic velocity gradients in the upper levels of layer 2 (e.g., Hyndman, 1974; Christensen et al., 1974) which originate from decreasing sea floor weathering with increasing depth. Since Site 396B is located in relatively young crust, a gradual increase in velocity with depth is not apparent (Figure 1). The variation of velocity and density within this basalt suite is primarily controlled by the variation in effective porosity (Figure 2), rather than degree of alteration. Basalts from Site 396B have slightly higher velocities for a given density than basalts from other DSDP sites (Christensen and Salisbury, 1975). Abundant phenocrysts, vesicles, or carbonate might cause this difference.

The geometric mean resistivity of 21 samples is 220 $\pm 40 \mathrm{ohm}-\mathrm{m}$, the values ranging from 36 to $630 \mathrm{ohm}-\mathrm{m}$. This mean is almost identical with that previously reported for 87 samples from holes into the MidAtlantic Ridge near $37^{\circ} \mathrm{N}$ (Hyndman and Drury, 1977) and for other sea floor tholeiites (e.g., Hyndman and Ade-Hall, 1974; Drury, 1976). The resistivity correlates well with porosity (Figure 3 ) indicating, as with

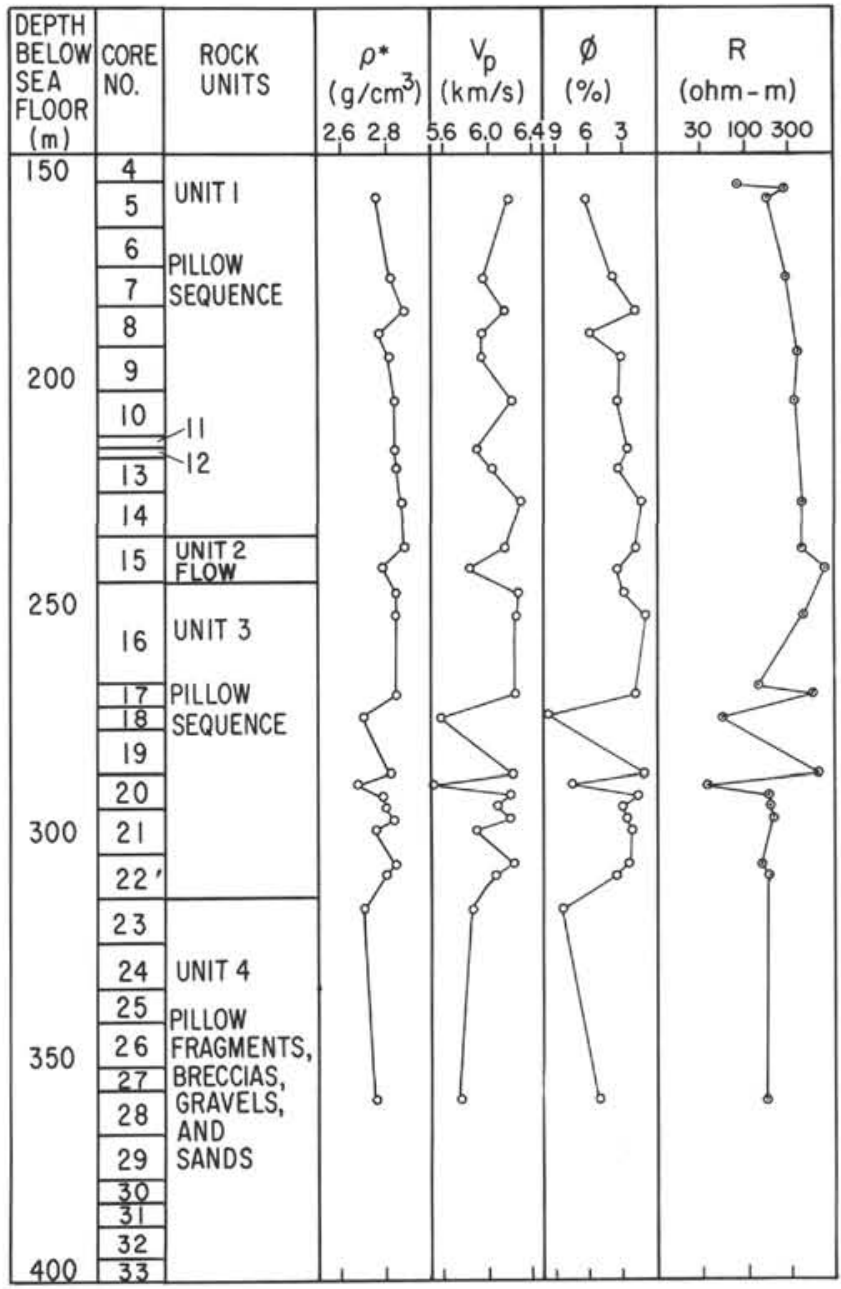

Figure 1. Variation of physical properties with depth into basement, Hole 396B.

previously measured basalts, that conduction is primarily through the seawater pore fluid. The slope of the relation $r$ about equal to 2 implies roughly equidimensional pore spaces rather than thin cracks (see Archie, 1942; Hyndman and Drury, 1977Y. Previous measurements on samples from DSDP Leg 37 suggested an even higher slope of about 2.5 , implying very poor interconnection of pore spaces in sea floor basalts, but the higher value is not substantiated by the limited new data. The downhole logging (R.J. Kirkpatrick, this volume), suggests an $r$ value of about 


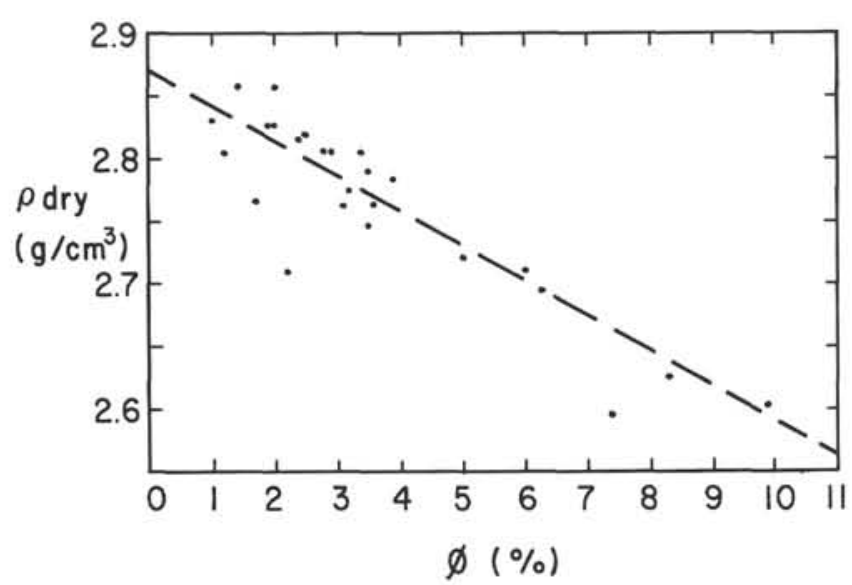

Figure 2. Variation of dry density with porosity for basalt samples from Hole $396 B$.

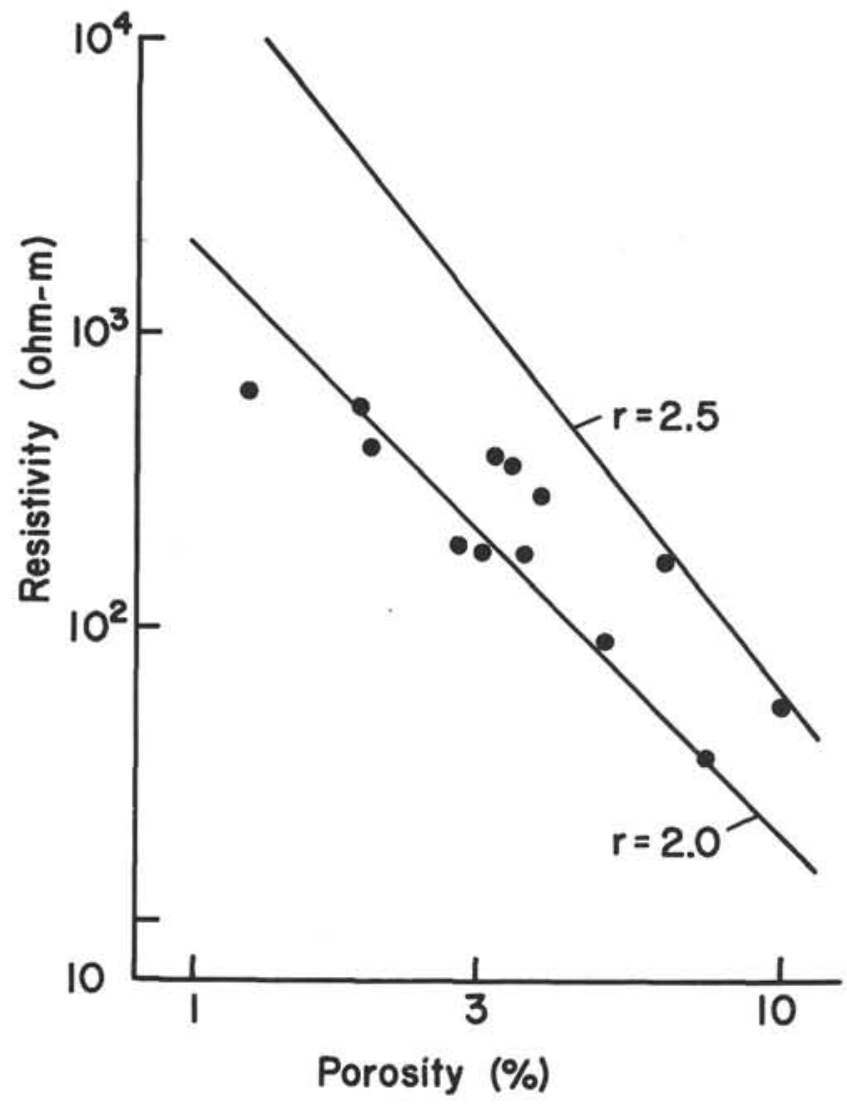

Figure 3. Variation of electrical resistivity with porosity for basalts from Hole 396B. The slope $r$ depends on the extent of interconnection of the pores spaces.
1.33. The mean larger scale resistivity from downhole logging requires at least 4 per cent of large-scale crack and void porosity in the more massive drilled sections, in addition to the average of 4.1 per cent porosity observed in the minicore samples. Most of the drilled section must have much more large-scale porosity.

\section{ACKNOWLEDGMENTS}

G. Bussod and J. Willemin assisted in the velocity measurements. Financial support for the velocity measurements was provided by the Office of Naval Research contract N-00014-75-C-0502 and by a grant from the Research Foundation of the State University of New York.

\section{REFERENCES}

Archie, G. E., 1942. The electrical resistivitylog as an aid in determining some reservoir characteristics: AIME Trans., v. $146,54-67$.

Birch, F., 1960. The velocity of compressional waves in rocks to 10 kilobars, 1: J. Geophys. Res., v. 65, p. 1083-1102. ,1961. The velocity of compressional waves in rocks to 10 kilobars, 2: J. Geophys. Res., v. 66, p. 2199-2224.

Christensen, N.I. and Salisbury, M.H., 1975. Structure and constitution of the lower oceanic crust: Rev. Geophys. Space Phys., v. 13, p. 57-86.

Christensen, N.I., Salisbury, M.H., Fountain, D.M., and Carlson, R.L., 1974. Velocities of compressional and shear waves in DSDP Leg 27 basalts. In Veever, J.J., Heirtzler, J.R., et al., Initial Reports of the Deep Sea Drilling Project, v. 27: Washington (U.S. Government Printing Office), p. 445-449.

Christensen, N.I. and Shaw, G.H., 1970. Elasticity of mafic rocks from the Mid-Atlantic Ridge: Roy. Astron. Soc. Geophys. Jr., v. 20, p. 271-284.

Drury, M.J., 1976. Electrical resistivity of basalts, DSDP Leg 34. In Hart, S.R., Yeats, R.S., et al., Initial Reports of the Deep Sea Drilling Project, v. 34: Washington (U.S. Government Printing Office), p. 549-552.

Hyndman, R.D., 1974. Seismic velocities of basalts from DSDP Leg 26. In Luyendyk, B. P., Davies, T. A., et al., Initial Reports of the Deep Sea Drilling Project, v. 26: Washington (U.S. Government Printing Office), p. 509512.

Hyndman, R.D. and J.M. Ade-Hall, 1974. Electrical resistivity of basalts from DSDP Leg 26. In Luyendyk, B.P., Davies, T.A., et al., Initial Reports of the Deep Sea Drilling Project, v. 26: Washington(U.S, Government Printing Office), p. 505-508.

Hyndman, R.D. and M.J. Drury, 1977. Physical properties of basalts, gabbros and ultramafic rocks from DSDP Leg 37 In Aumento, F., Melson, W.G., et al., Initial Reports of the the Deep Sea Drilling Project, v. 39: Washington (U.S. Government Printing Office), p. 395-402. 